\title{
Phosphorylation and acetylation modifications of FOXO3a: Independently or synergistically? (Review)
}

\author{
XIANWANG WANG ${ }^{1}$, SHUJUAN HU ${ }^{2}$ and LEI LIU $^{3}$ \\ ${ }^{1}$ Laboratory of The Neuronal Network and Brain Diseases Modulation, School of Medicine; \\ ${ }^{2}$ Institute of Physical Education, Yangtze University, Jingzhou, Hubei 434023; ${ }^{3}$ MOE Key Laboratory \\ of Laser Life Science and Institute of Laser Life Science, College of Biophotonics, South \\ China Normal University, Guangzhou, Guangdong 510631, P.R. China
}

Received August 19, 2015; Accepted January 26, 2016

DOI: $10.3892 / \mathrm{ol} .2017 .5851$

\begin{abstract}
Forkhead box class O 3a (FOXO3a) is a transcription factor that has emerged as being a tumor suppressor and longevity factor. The precise regulation of FOXO3a transactivation of target genes is achieved via post-translational modifications (PTMs) and specific protein-protein interactions. The multiple types of PTMs that FOXO3a undergoes, including phosphorylation, acetylation, methylation and ubiquitination, serve important roles in directing its subcellular localization and transcription activity, which are central to the integration of insulin/growth factor signaling and oxidative/nutrient stress signaling. The present review summarizes the modifications of FOXO3a that occur via phosphorylation and acetylation. In addition, the synergistic effect of multiple
\end{abstract}

Correspondence to: Dr Xianwang Wang, Laboratory of Neuronal Network and Brain Disease Modulation, School of Medicine, Yangtze University, No. 1 South Circle Road, Jingzhou, Hubei 434023, P.R. China

E-mail: 275379987@qq.com

Dr Lei Liu, MOE Key Laboratory of Laser Life Science and Institute of Laser Life Science, College of Biophotonics, South China Normal University, No. 55 Zhongshan Road West, Guangzhou, Guangdong 510631, P.R. China

E-mail: 281514217@qq.com

Abbreviations: FOXO3a, forkhead box class O 3a; FH, forkhead DNA-binding domain; NLS, nuclear localization signal; NES, nuclear export signal; CTD, C-terminal transactivation domain; FRE, forkhead response element; PTM, post-translational modification; CK1, casein kinase-1; HAT, histone acetyltransferase; SGK, serum and glucocorticoid-induced kinase; ERK, extracellular signal-regulated kinase; AMPK, AMP-activated protein kinase; IKK, IкB kinase; MST1, mammalian sterile 20-like kinase 1; LMTK3, lemur tyrosine kinase-3; DYRK1, dual-specificity tyrosine phosphorylated and regulated kinase 1 ; CDK1/2, cyclin-dependent kinase $1 / 2$; SIRT, silent information regulator; $\mathrm{CR}$, conserved region

Key words: FOXO3a, phosphorylation, acetylation, SIRT1, transactivation phosphorylations on FOXO3a and the crosstalk between phosphorylation and acetylation in the regulation of FOXO3a are discussed. These discussions may highlight potential strategies for the prevention of cancer and aging.

\section{Contents}

1. Introduction

2. Regulation of FOXO3a by phosphorylation

3. Regulation of FOXO3a by acetylation

4. Mechanisms of phosphorylation and acetylation of FOXO3a

5. Conclusion

\section{Introduction}

Forkhead box O class (FOXO) proteins are evolutionally conserved transcription factors. FOXO transcription factors perform an important role in tumor suppression, longevity and metabolism by up regulating target genes associated with oxidative stress resistance, metabolism, apoptosis, cell cycle arrest, aging and autophagy (1-5). There are four FOXOs members in mammalian cells, FOXO1, 3, 4 and 6 (6). As presented in Fig. 1, all FOXO members consist of the following four conserved regions (CRs): An N-terminal highly conserved 100-residue-long forkhead DNA-binding domain (FH); a nuclear localization signal (NLS); a nuclear export signal (NES); and a C-terminal transactivation domain (CTD) (1,7-9). Crystal structure analysis revealed that the winged helix $\mathrm{H} 3$ region of the $\mathrm{FH}$ is the primary element for DNA recognition $(7,8)$ and is capable of binding the consensus DNA sequence, 5'-TTGTTTAC-3', termed the forkhead response element (FRE) $(9,10)$.

There are two aspects of FOXO regulation; subcellular localization and transcription activity. Multiple post-translational modifications (PTMs) serve a major role in determining FOXO subcellular localization and transcription activity. As shown in Fig. 2, in response to insulin/growth factor signaling, a number of different protein kinases, including protein kinase B (AKT), serum and glucocorticoid-induced kinase (SGK), extracellular signal-regulated kinase (ERK), 
casein kinase-1 (CK1), and IkB kinase (IKK), are capable of inducing translocation of FOXO into the cytoplasm (11-13). By contrast, several upstream regulators, such as c-Jun N-terminal kinase (JNK), mammalian sterile 20-like kinase 1 (MST1), AMP-activated protein kinase (AMPK) and lemur tyrosine kinase-3 (LMTK3) can phosphorylate FOXO at specific residues, which in turn promotes nuclear localization and transcriptional activity $(11,12,14,15)$. In addition to various phosphorylation modifications, acetylation is another important regulator of FOXO proteins. The cyclic AMP response element binding protein (CBP) and its associated protein $\mathrm{p} 300$ form a coactivator complex (CBP/p300), which possesses histone acetyl transferase (HAT) activity, interacts with FOXO and regulates FOXO transcriptional activity $(16,17)$. Silent information regulator (SIRT) 1 and 2 are histone deacetylases, which deacetylate FOXO and increase the expression of specific stress resistance genes, including $\beta$-catenin and Gadd45 (18,19).

The effect of different types of phosphorylation on the function of FOXO3a has been studied extensively (19). This has revealed that phosphorylation and acetylation frequently occur simultaneously and affect one other in certain conditions (19). The present review summarized the phosphorylation and acetylation sites of FOXO3a, in addition to analyzing the reciprocal regulation of phosphorylation/acetylation and their effects on FOXO3a subcellular localization and transcriptional activity.

\section{Regulation of FOXO3a by phosphorylation}

Phosphorylation modification regulates FOXO activity via a cytoplasmic-nuclear shuttle mechanism. FOXO3a is targeted for phosphorylation by numerous protein kinases. Several regulators have been identified as kinases of FOXO3a in mammals (Table I). A summary of these regulatory mechanisms are described below.

Negative effect of phosphorylation on FOXO3a. As the major regulator of FOXO activation, AKT is responsible for the phosphorylation of FOXO3a at threonine (Thr)32, serine (Ser)253 and Ser315. This phosphorylation leads to the exclusion of FOXO3a from the nucleus and the association of FOXO3a with 14-3-3 proteins, which subsequently inhibits the transactivation activity of FOXO3a $(20,21)$. In addition to phosphorylation and inhibition by AKT, FOXO3a is phosphorylated by a number of other kinases. SGK is reported to share the same FOXO phosphorylation sites as AKT, and also decreases FOXO3a nuclear localization and DNA-binding activity $(11,22)$. ERKs have been demonstrated to inhibit FOXO3a by directly phosphorylating FOXO3a at Ser294, Ser344 and Ser425, and to induce the degradation of FOXO3a via the murine double minute-2 signaling pathway, which consequently stimulates cell survival and anti-apoptotic gene expression (23). In addition, IKK can phosphorylate FOXO3a at Ser644, promoting cytoplasmic retention and polyubiquitination-mediated degradation of FOXO3a (13).

CK1 has been identified to phosphorylate FOXO3a at Ser318 and Ser321, leading to nuclear export and the inhibition of FOXO3a activity (24). However, the phosphorylation of FOXO3a at Ser315 by AKT is required for CK1-dependent Ser318 and Ser321 phosphorylation (24). Dual-specificity tyrosine phosphorylation and regulated kinase 1 (DYRK1) is also able to phosphorylate FOXO proteins. It has been reported that DYRK1A phosphorylates FOXO1 at Ser329 and FOXO3a at Ser325 $(1,25)$. DYRK1B serves an important role in ovarian cancer cell survival by mediating the nuclear translocation of FOXO3a and the expression of apoptotic genes (26). These data suggest that the phosphorylation of FOXO3a by these kinases induces cytoplasmic retention and degradation.

Positive effect of phosphorylation on FOXO3a. FOXOactivating kinases can promote the nuclear translocation and activity of FOXO3a. JNK is a member of the oxidative stress-activated mitogen-activated protein kinase family. Upon activation, JNK can phosphorylate Ser574 of FOXO3a, which antagonizes the AKT signaling pathway and promotes the transcription and nuclear translocation of FOXO3a $(4,27)$. A second kinase capable of promoting FOXO3a activation is AMPK, which can phosphorylate FOXO3a at Thr179, Ser399, Ser413, Ser555, Ser588 and Ser626 (28). AMPK phosphorylation increases the transcription activity of FOXO3a without effecting the cytoplasmic-nuclear shuttling of FOXO3a (28). The potential molecular mechanism through which AMPK regulates FOXO is the recruitment of additional proteins to transcriptional complexes or the direction of FOXO to certain promoter regions. In response to doxorubicin, p38 phosphorylates FOXO3a at Ser7, which promotes FOXO3 a nuclear localization in breast carcinoma MCF-7 cells (29).

MST1 is also a positive regulator of FOXO activity. Activated MST1 interacts with FOXO3a to phosphorylate Ser207, which inhibits the association of FOXO3a with 14-3-3 proteins, and promotes the nuclear translocation and transcription activity of FOXO3a (15). LMTK3 is a serine-threonine-tyrosine kinase that can inhibit protein kinase $\mathrm{C}$ and $\mathrm{AKT}$, thereby promoting the binding of $\mathrm{FOXO} 3$ a to the estrogen receptor 1 gene promoter element (30). In addition, upon silencing of LMTK3, the level of AKT phosphorylation at Ser473 is increased, and the frequency of FOXO3a phosphorylation by AKT at Ser318, Ser321 and Thr32 is markedly decreased (30). Cyclin-dependent kinase 1/2 (CDK1/2) has been demonstrated to phosphorylate FOXO1 at conserved sites (31), although it remains unclear whether they phosphorylate FOXO3a.

There is disagreement regarding the effect of CDK1/2 on FOXO1. CDK1 has been demonstrated to elicit cellular proliferation, survival and tumorigenesis via the specific phosphorylation of FOXO1 at Ser249, which inhibits the activity of FOXO1 (31). In addition, CDK2 has been reported to phosphorylate FOXO1 at Ser249 and Ser298, causing cytoplasmic accumulation and inhibiting FOXO1 activity (32). However, a previous study demonstrated that the phosphorylation of FOXO1 at Ser249 disrupted the binding between FOXO1 and 14-3-3 proteins, thereby promoting the nuclear accumulation of FOXO1 and FOXO1-dependent transcription, leading to cell death in neurons (33). Similarly to FOXO1, FOXO3a has been suggested as a potential target of CDK1/2. Thus, studies investigating the effect of CDK1/2 on the subcellular localization and transcription activity of FOXO3a in response to certain stimuli are warranted.

Synergistic effect of multiple phosphorylations of FOXO3a. The effects of multiple phosphorylation modifications of 
Table I. FOXO3a kinases, their phosphorylation sites and effects.

\begin{tabular}{lllll}
\hline Kinase & FOXO3a type & \multicolumn{1}{c}{ Sites of phosphorylation } & Localization & Effect \\
\hline ERK/p38 & Human & $\mathrm{S}^{7}, \mathrm{~S}^{12}, \mathrm{~S}^{284}, \mathrm{~S}^{294}, \mathrm{~S}^{325}, \mathrm{~S}^{344}, \mathrm{~S}^{425}, \mathrm{~S}^{487}$ & Cytoplasmic & Inhibition and degradation \\
& Rat & $\mathrm{S}^{7}, \mathrm{~S}^{12}, \mathrm{~S}^{283}, \mathrm{~S}^{293}$ & Cytoplasmic & Inhibition and degradation \\
& Mouse & $\mathrm{S}^{7}, \mathrm{~S}^{12}, \mathrm{~S}^{283}, \mathrm{~S}^{293}, \mathrm{~S}^{424}$ & Cytoplasmic & Inhibition and degradation \\
IKK & Human & $\mathrm{S}^{644}$ & Cytoplasmic & Inactivation and degradation \\
AKT/SGK & Human & $\mathrm{T}^{32}, \mathrm{~S}^{253}, \mathrm{~S}^{315}$ & Cytoplasmic & Inhibition \\
& Rat & $\mathrm{T}^{32}, \mathrm{~S}^{252}$ & Cytoplasmic & Inhibition \\
& Mouse & $\mathrm{T}^{32}, \mathrm{~S}^{252}, \mathrm{~S}^{314}$ & Cytoplasmic & Inhibition \\
CK1 & Human & $\mathrm{S}^{318}, \mathrm{~S}^{321}$ & Cytoplasmic & Inhibition \\
& Mouse & $\mathrm{S}^{317}, \mathrm{~S}^{320}$ & Cytoplasmic & Inhibition \\
DYRK1A/B & Human & $\mathrm{S}^{325}$ & Cytoplasmic & Inhibition \\
AMPK & Human & $\mathrm{T}^{179}, \mathrm{~S}^{399}, \mathrm{~S}^{413}, \mathrm{~S}^{555}, \mathrm{~S}^{588}, \mathrm{~S}^{626}$ & No effect & Transactivation \\
LMTK3 & Mouse & $\mathrm{S}^{412}$ & No effect & Transactivation \\
& Human & $\mathrm{S}^{318}, \mathrm{~S}^{321}$ & Nuclear & Transactivation \\
MST1 & Mouse & $\mathrm{S}^{317}, \mathrm{~S}^{320}$ & Nuclear & Transactivation \\
JNK & Human & $\mathrm{S}^{209}, \mathrm{~S}^{215}, \mathrm{~S}^{231}, \mathrm{~S}^{232}$ & Nuclear & Activation \\
& Mouse & $\mathrm{S}^{208}$ & Nuclear & Activation
\end{tabular}

FOXO3a, forkhead box class O 3a; S, serine; T, threonine; ERK, extracellular signal-regulated kinase; IKK, IkB kinase; AKT, protein kinase B; SGK, serum and glucocorticoid-induced kinase; CK1, casein kinase-1; DYRK1, dual-specificity tyrosine phosphorylation and regulated kinase 1; AMPK, AMP-activated protein kinase; LMTK3, lemur tyrosine kinase-3; MST1, mammalian sterile 20-like kinase 1; JNK, c-Jun N-terminal kinase.

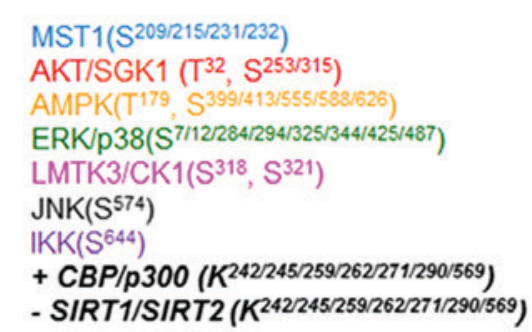

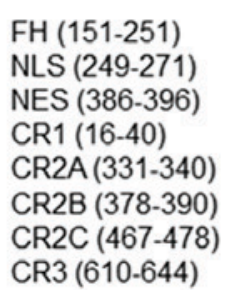

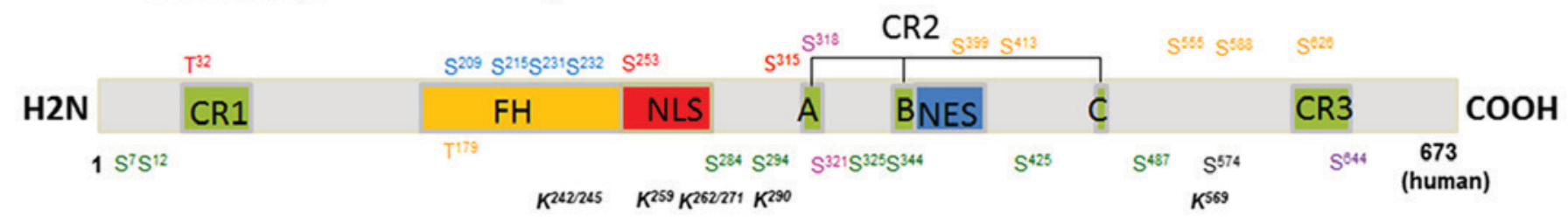

Figure 1. Amino acid sites of phosphorylation and acetylation of human FOXO3a. The different colors represent different kinases for FOXO3a modification and different regions of FOXO3a protein. A, B and C represent different sections of CR2. MST1, mammalian sterile 20-like kinase 1; AKT, protein kinase B; SGK, serum and glucocorticoid-induced kinase; AMPK, AMP-activated protein kinase; ERK, extracellular signal-regulated kinase; LMTK, lemur tyrosine kinase; CK1, casein kinase-1; JNK, c-Jun N-terminal kinase; IKK, IкB kinase; CBP, cyclic AMP response element binding protein; SIRT, silent information regulator; CR, conserved region; FH, forkhead; NLS, nuclear localization signal; NES, nuclear export signal; S, serine; T, threonine; K, lysine.

FOXO3a by different kinases are not independent. Specific residues of FOXO3a can be phosphorylated by a number of kinases. As illustrated in Table I, ERK and p38 share several conserved FOXO3a phosphorylation sites in humans, rats and mice. Similarly, AKT and SGK1 have several of the same phosphorylation sites on FOXO3a, and inhibit the activity of FOXO3a. In humans, two conserved residues sites (Ser318 and Ser321) can be phosphorylated by LMTK3 and CK1. However, LMTK3 and CK1 can elicit opposite effects through phosphorylating the same sites. LMTK3 phosphorylation of FOXO3a at Ser318/Ser321 in humans has been demonstrated promote FOXO3a activity $(30,31)$. By contrast, the phosphorylation of these two sites by $\mathrm{CK} 1$ results in the inhibition of FOXO3a activity $(11,24)$.

Under certain conditions, kinases are likely to simultaneously regulate one other. As described above, CK1 phosphorylates FOXO3a at Ser318/Ser321 following the phosphorylation of Ser315 by AKT, which enabled the 


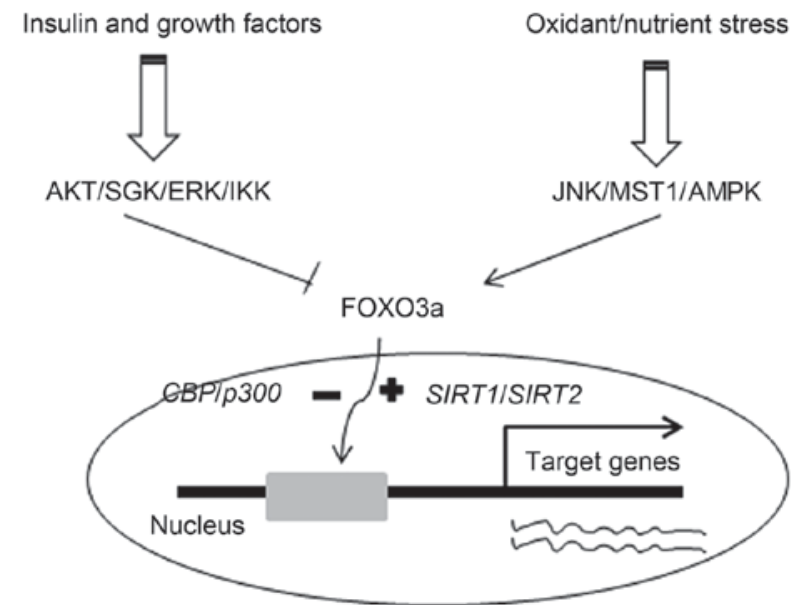

Figure 2. Association between insulin/growth factor signaling and oxidative/ nutrient stress signaling in regards to FOXO3a. AKT, protein kinase B; SGK, serum and glucocorticoid-induced kinase; ERK, extracellular signal-regulated kinase; IKK, IкB kinase; JNK, c-Jun N-terminal kinase; MST1, mammalian sterile 20-like kinase 1; AMPK, adenosine monophosphate-activated protein kinase; $\mathrm{CBP}$, cyclic AMP response element binding protein; FOXO3a, forkhead box class O 3a; SIRT, silent information regulator.

identification of consensus sequences for CK1 phosphorylation (24). Under oxidative stress, JNK and MST1 participate in similar signaling pathways and act in a complementary manner to activate FOXO3a. JNK directly phosphorylates FOXO3a and also phosphorylates MST1 at Ser82, which leads to enhanced MST1 activation (34). Accordingly, activated MST1 phosphorylates FOXO3 at Ser207 and promotes cell death (34). AKT inhibits MST1 through phosphorylating it at Thr120, blocking MST1-mediated phosphorylation and activation of FOXO3a (35). Additionally, MST1 has also been demonstrated to be capable of binding to and inhibiting AKT, resulting in an inhibition of cellular proliferation (36).

\section{Regulation of FOXO3a by acetylation}

Different PTMs change the subcellular localization and/or DNA-binding activity of FOXO3a, allowing FOX3a to serve a role in various cellular activities. Similarly to phosphorylation, acetylation is another PTM that can regulate FOXO3a.

Dual effects of CBP/p300-mediate acetylation of FOXO3a. In the majority of cases, acetylation aids the transactivation of genes. The binding of the $\mathrm{CBP} / \mathrm{p} 300$ coactivator to FOXO proteins is essential for the transactivation of target genes (37). CBP/p300, which acetylates nucleosomal histones, can also acetylate FOXO3a. Acetylation of nucleosomal histones stimulates transcription, whereas the acetylation of FOXO3a has been reported to attenuate its transcriptional activity $(38,39)$. It is possible that acetylated FOXO3a has a decreased DNA-binding activity. In addition, acetylation has been observed to cause the stimulation and depression of FOXO protein activity, dependent on the FOXO isoform and binding partners present $(38,39)$. A previous study demonstrated that $\mathrm{CBP} / \mathrm{p} 300$ acetylation of $\mathrm{FOXO1}$ at specific highly conserved sites decreased DNA-binding activity (38). Additionally, under oxidative stress CBP has been demonstrated to trigger the acetylation of FOXO4 and inhibit its activity (39).
Furthermore, FOXO3a interacts with the HAT p300, the acetylation of which causes cytoplasmic retention and induces the degradation of FOXO3a via the proteasome $(40,41)$. In response to nutrient deprivation, dominant-negative p300 was identified to induce FOXO3a nuclear translocation, while transfection of $\mathrm{CBP} / \mathrm{p} 300$ resulted in FOXO3a repression (41). In addition, AKT has been demonstrated to phosphorylate acetylated FOXO proteins, which resulted in cytoplasmic retention and 14-3-3 proteins binding (42).

Deacetylation of FOXO3a is induced by sirtuins. The $\mathrm{CBP} / \mathrm{p} 300$-mediated acetylation of FOXO3a attenuates its transcriptional activity. By contrast, deacetylation by SIRT1 increases the activity of FOXO3a. It has been reported that SIRT1 deacetylates and activates FOXO3a in the nucleus accumbens, leading to the expression of several targets genes (43). However, the deacetylation of FOXO3a by SIRT1 has also been reported to repress FOXO3a activity. Motta et al (44) demonstrated that SIRT1 deacetylates and decreases the activity of FOXO proteins, including FOXO1, FOXO3a and FOXO4. A recent study also demonstrated that SIRT1 could bind to and deacetylate FOXO3a, which increased its ubiquitination level and protected against apoptosis (45). SIRT2 also serves an important role in FOXO3a deacetylation. SIRT2 deacetylates FOXO3, increasing the DNA-binding activity of FOXO3 and thus increasing the expression of its target genes, cyclin-dependent kinase inhibitor 1B (p27Kip1), manganese superoxide dismutase and BCL2 like 11 (Bim) $(18,19)$. Notably, in response to oxidative stress, SIRT1 interacts with and deacetylates FOXO3a, which decreases the expression of pro-apoptotic Bim, but increases the expression of cell cycle arrest genes, including p27Kip1 and growth arrest and DNA inducible $\alpha$ (16). These results highlight the complex mechanisms by which FOXO is regulated by acetylation and deacetylation. It is possible that the interaction between acetylation and phosphorylation in FOXO regulation may be the result of further mechanisms that have not yet been elucidated.

\section{Mechanisms of phosphorylation and acetylation of FOXO3a}

As shown in Fig. 1, FOXO3a consists of the following CRs: A FH (residues 151-251); a NLS (residues 249-271); a NES (residues 386-396); CR1 (residues 16-40); CR2A (residues 331-340); CR2B (residues 378-390); CR2C (residues 467-478); and CR3 (residues 610-650) $(1,7-10,19,46)$. FH is a conserved DNA-binding domain, which is capable of binding to the FRE and allowing transcription (1,7-9). MST1 (Ser209, Ser215, Ser231 and Ser232) and AMPK (Thr179) can directly phosphorylate FOXO3a in the FH region $(15,34,43)$. These conserved phosphorylation sites have been demonstrated to increase the DNA-binding activity of FOXO3a. NLS and NES domains are important for FOXO3a subcellular localization $(15,34,43)$. AKT/SGK phosphorylates FOX3a at Ser253, which is located in the NLS and acts to disrupt NLS activity by introducing a negative charge to the basic NLS region (19). Phosphorylation of Ser315 reveals the NES of FOXO, increasing its rate of nuclear export (38). The CR3 domain is a CTD that can interact with the FH region $(7,10)$. It has been 
demonstrated that the binding of $\mathrm{FH}$ to FRE releases CR3, allowing it to bind to the KIX domain of $\mathrm{CBP} / \mathrm{p} 300$, which can then acetylate and inhibit the DNA binding capacity of FOXO3a (36). CR2C is also a transactivation domain, and mediates the association between FOXO3a and CBP/p300 by binding to the KIX domain $(10,46)$.

Under certain conditions, coordinating the regulation of phosphorylation and acetylation may synergistically control the function of FOXO3a, including DNA binding activity, subcellular localization and transactivation activity. The processes of acetylation and phosphorylation of the CRs of FOXO3a affects one other. It is known that MST1 (Ser209, Ser215, Ser231 and Ser232) and AMPK (Thr179) can directly phosphorylate FOXO3a in the FH domain, which increases the DNA-binding affinity of FOXO3a. Activated SIRT1 deacetylates Lysine (Lys)242 and Lys245 in the FH domain and also enhances the DNA binding of FOXO3a (43). Previous studies have demonstrated that SIRT1, MST1 and AMPK are activated in response to oxidative stress $(15,16,28,34,35)$. This suggests that the DNA binding affinity of FOXO3a is controlled by the interaction between acetylation and phosphorylation.

Certain acetylation sites in FOXO3a surround a consensus site for AKT-induced Ser phosphorylation within the NLS domain. Acetylation of FOXO3a by $\mathrm{CBP} / \mathrm{p} 300$ has been suggested to promote AKT-mediated phosphorylation at Ser253 in the NLS motif $(41,42)$. It is therefore possible that the acetylation of Lys259, Lys262 and Lys271 in the NLS region of FOXO3a changes the conformation of the NLS and results poor affinity for the DNA, making the NLS prone to phosphorylation by AKT (42). AKT phosphorylation of FOXO3a at Thr32 in the CR1 region and Ser253 in the NLS region increases the binding of FOXO3a to 14-3-3 proteins, and decreases the interaction between FOXO3a and CBP/p300 (42). Phosphorylated FOXO3a (Thr32/Ser253) is unable to bind to CBP/ p300 and loses the ability for chromatin remodeling. AMPK phosphorylation of FOXO3a at Ser626 in the CR3 domain increases the binding of $\mathrm{CBP} / \mathrm{p} 300$ and transactivation activity of FOXO3a (10). It has also been demonstrated that AMPK enhances SIRT1 activity by increasing cellular NAD ${ }^{+}$levels, resulting in the deacetylation of FOXO3a (47). The effect of IKK-mediated Ser644 phosphorylation in the acetylated CR3 region of FOXO3a remains unclear. Thus, the potential interaction between acetylation and phosphorylation in the conserved PTM sites of FOXO3a discussed in the present review is an important area to investigate in future studies.

\section{Conclusion}

In conclusion, the effect of phosphorylation and acetylation on the transactivation activity of FOXO3a represents a complex balance between insulin/growth factor signaling and oxidative stress signaling (Fig. 2). Insulin and growth factors initiate a signaling cascade that results in the activation of kinases, including AKT/SGK, ERK and IKK (11-13). These kinases phosphorylate FOXO3a at specific sites, inducing its cytoplasmic retention and/or degradation, leading to inhibition of target genes transcription $(13,19-23)$. By contrast, oxidative or nutrient stress signals induce activation of JNK, MST1 and AMPK, which phosphorylate FOXO3a at the conserved residue sites, resulting in nucleus localization of FOXO3a and promoting the transcription of target genes $(11,12,14,15)$. Besides, stresses also increase SIRT1/SIRT2-FOXO3a interaction, resulting in tightly binding of FOXO3a to DNA binding domain and enhancing target genes transcription $(18,19,45)$. Although acetylation does not always correspond with phosphorylation, phosphorylation and acetylation can cooperate to regulate FOXO3a in response to different stimuli. Thus, the potent functions of FOXO3a are tightly controlled by complex signaling pathways under physiological conditions. Gaining better understanding of the PTMs of FOXO3a remains an important area of investigation and should be addressed in future studies.

\section{Acknowledgements}

The present review was supported by Hubei Province Natural Science Foundation of China (grant no. 2016CFB180), Hubei Province Health and Family Planning Scientific Research Project (grant no. WJ2016Y07), Jingzhou Science and Technology Development Planning Project (grant no. JZKJ15063) and the National Natural Science Foundation of China (grant no. 31470072).

\section{References}

1. Calnan DR and Brunet A: The FoxO code. Oncogene 27: 2276-2288, 2008.

2. Wang J, Liu S, Yin Y, Li M, Wang B, Yang L and Jiang Y: FOXO3-mediated up-regulation of Bim contributes to rhein-induced cancer cell apoptosis. Apoptosis 20: 399-409, 2015.

3. Kloet DE and Burgering BM: The PKB/FOXO switch in aging and cancer. Biochim Biophys Acta 1813: 1926-1937, 2011.

4. Wang XW, Chen WR and Xing D: A pathway from JNK through decreased ERK and Akt activities for FOXO3a nuclear translocation in response to UV irradiation. J Cell Physiol 227: 1168-1178, 2012.

5. Zhou J, Liao W, Yang J, Ma K, Li X, Wang Y, Wang D, Wang L, Zhang Y, Yin Y, et al: FOXO3 induces FOXO1-dependent autophagy by activating the AKT1 signaling pathway. Autophagy 8: 1712-1723, 2012.

6. Wang M, Zhang X, Zhao H, Wang Q and Pan Y: FoxO gene family evolution in vertebrates. BMC Evol Biol 9: 222, 2009.

7. Clark KL, Halay ED, Lai E and Burley SK: Co-crystal structure of the HNF-3/fork head DNA-recognition motif resembles histone H5. Nature 364: 412-420, 1993.

8. Obsil T and Obsilova V: Structural basis for DNA recognition by FOXO proteins. Biochim Biophys Acta 1813: 1946-1953, 2011.

9. Wang F, Marshall CB, Yamamoto K, Li GY, Plevin MJ, You H, Mak TW and Ikura M: Biochemical and structural characterization of an intramolecular interaction in FOXO3a and its binding with p53. J Mol Biol 384: 590-603, 2008.

10. Wang F, Marshall CB, Yamamoto K, Li GY, Gasmi-Seabrook GM, Okada H, Mak TW and Ikura M: Structures of KIX domain of $\mathrm{CBP}$ in complex with two FOXO3a transactivation domains reveal promiscuity and plasticity in coactivator recruitment. Proc Natl Acad Sci USA 109: 6078-6083, 2012.

11. Boccitto $M$ and Kalb RG: Regulation of foxo-dependent transcription by post-translational modifications. Curr Drug Targets 12: 1303-1310, 2011.

12. Zhao Y, Wang Y and Zhu WG: Applications of post-translational modifications of FoxO family proteins in biological functions. J Mol Cell Biol 3: 276-282, 2011.

13. Hu MC, Lee DF, Xia W, Golfman LS, Ou-Yang F, Yang JY, Zou Y, Bao S, Hanada N, Saso H, et al: IkappaB kinase promotes tumorigenesis through inhibition of forkhead FOXO3a. Cell 117: 225-237, 2004.

14. Essers MA, Weijzen S, de Vries-Smits AM, Saarloos I, de Ruiter ND, Bos JL and Burgering BM: FOXO transcription factor activation by oxidative stress mediated by the small GTPaseRal and JNK. EMBO J 23: 4802-4812, 2004. 
15. Lehtinen MK, Yuan Z, Boag PR, Yang Y, Villén J, Becker EB, DiBacco S, de la Iglesia N, Gygi S, Blackwell TK and Bonni A: A conserved MST-FOXO signaling pathway mediates oxidative-stress responses and extends life span. Cell 125: 987-1001, 2006.

16. Brunet A, Sweeney LB, Sturgill JF, Chua KF, Greer PL, Lin Y, Tran H, Ross SE, Mostoslavsky R, Cohen HY, et al: Stress-dependent regulation of FOXO transcription factors by the SIRT1 deacetylase. Science 303: 2011-2015, 2004.

17. Daitoku H, Sakamaki J and Fukamizu A: Regulation of FoxO transcription factors by acetylation and protein-protein interactions. Biochim Biophys Acta 1813: 1954-1960, 2011.

18. Nakagawa T and Guarente L: Sirtuins at a glance. J Cell Sci 124 833-838, 2011.

19. Wang F, Nguyen M, Qin FX and Tong Q: SIRT2 deacetylates FOXO3a in response to oxidative stress and caloric restriction. Aging Cell 6: 505-514, 2007.

20. Brunet A, Bonni A, Zigmond MJ, Lin MZ, Juo P, Hu LS, Anderson MJ, Arden KC, Blenis J and Greenberg ME: Akt promotes cell survival by phosphorylating and inhibiting a Forkhead transcription factor. Cell 96: 857-868, 1999.

21. Dobson M, Ramakrishnan G, Ma S, Kaplun L, Balan V, Fridman $\mathrm{R}$ and Tzivion G: Bimodal regulation of FoxO3 by AKT and 14-3-3. Biochim Biophys Acta 1813: 1453-1464, 2011

22. Brunet A, Park J, Tran H, Hu LS, Hemmings BA and Greenberg ME: Protein kinase SGK mediates survival signals by phosphorylating the forkhead transcription factor FKHRL1 (FOXO3a). Mol Cell Biol 21: 952-965, 2001.

23. Yang JY, Zong CS, Xia W, Yamaguchi H, Ding Q, Xie X, Lang JY, Lai CC, Chang CJ, Huang WC, et al: ERK promotes tumorigenesis by inhibiting FOXO3a via MDM2-mediated degradation. Nat Cell Biol 10: 138-148, 2008.

24. Rena G, Woods YL, Prescott AR, Peggie M, Unterman TG, Williams MR and Cohen P: Two novel phosphorylation sites on FKHR that are critical for its nuclear exclusion. EMBO J 21 2263-2271, 2002.

25. Woods YL, Rena G, Morrice N, Barthel A, Becker W, Guo S, Unterman TG and Cohen P: The kinase DYRK1A phosphorylates the transcription factor FKHR at Ser329 in vitro, a novel in vivo phosphorylation site. Biochem J 355: 597-607, 2001.

26. Gao J, Yang X, Yin P, Hu W, Liao H, Miao Z, Pan C and Li N The involvement of FoxO in cell survival and chemosensitivity mediated by Mirk/Dyrk1B in ovarian cancer. Int J Oncol 40 : $1203-1209,2012$

27. Tikhanovich I, Kuravi S, Campbell RV, Kharbanda KK, Artigues A, Villar MT and Weinman SA: Regulation of FOXO3 by phosphorylation and methylation in hepatitis $\mathrm{C}$ virus infection and alcohol exposure. Hepatology 59: 58-70, 2014.

28. Greer EL, Oskoui PR, Banko MR, Maniar JM, Gygi MP, Gygi SP and Brunet A: The energy sensor AMP-activated protein kinase directly regulates the mammalian $\mathrm{FOXO} 3$ transcription factor. J BiolChem 282: 30107-30119, 2007.

29. Ho KK, McGuire VA, Koo CY, Muir KW, de Olano N, Maifoshie E, Kelly DJ, McGovern UB, Monteiro LJ, Gomes AR, et al: Phosphorylation of FOXO3a on Ser-7 by p38 promotes its nuclear localization in response to doxorubicin. J Biol Chem 287: 1545-1555, 2012

30. Giamas G, Filipović A, Jacob J, Messier W, Zhang H, Yang D, Zhang W, Shifa BA, Photiou A, Tralau-Stewart C, et al: Kinome screening for regulators of the estrogen receptor identifies LMTK3 as a new therapeutic target in breast cancer. Nat Med 17: 715-719, 2011.

31. Liu P, Kao TP and Huang H: CDK1 promotes cell proliferation and survival via phosphorylation and inhibition of FOXO1 transcription factor. Oncogene 27: 4733-4744, 2008.
32. Huang H, Regan KM, Lou Z, Chen J and Tindall DJ: CDK2-dependent phosphorylation of FOXO1 as an apoptotic response to DNA damage. Science 314: 294-297, 2006.

33. Yuan Z, Becker EB, Merlo P, Yamada T, DiBacco S, Konishi Y, Schaefer EM and Bonni A: Activation of FOXO1 by Cdk1 in cycling cells and postmitotic neurons. Science 319: 1665-1668, 2008.

34. Bi W, Xiao L, Jia Y, Wu J, Xie Q, Ren J, Ji G and Yuan Z: c-Jun N-terminal kinase enhances MST1-mediated pro-apoptotic signaling through phosphorylation at serine 82. J Biol Chem 285: 6259-6264, 2010

35. Yuan Z, Kim D, Shu S, Wu J, Guo J, Xiao L, Kaneko S, Coppola D and Cheng JQ: Phosphoinositide 3-kinase/Akt inhibits MST1-mediated pro-apoptotic signaling through phosphorylation of threonine 120. J Biol Chem 285: 3815-3824, 2010.

36. Chao Y, Wang Y, Liu X, Ma P, Shi Y, Gao J, Shi Q, Hu J, Yu R and Zhou X: Mst1 regulates glioma cell proliferation via the AKT/mTOR signaling pathway. J Neurooncol 121: 279-288, 2015.

37. van der Heide LP and Smidt MP: Regulation of FoxO activity by CBP/p300-mediated acetylation. Trends Biochem Sci 30: 81-86, 2005.

38. Daitoku H, Hatta M, Matsuzaki H, Aratani S, Ohshima T, Miyagishi M, Nakajima T and Fukamizu A: Silent information regulator 2 potentiates Foxo1-mediated transcription through its deacetylase activity. Proc Natl Acad Sci USA 101: 10042-10047, 2004.

39. van der Horst A, Tertoolen LG, de Vries-Smits LM, Frye RA, Medema RH and Burgering BM: FOXO4 is acetylated upon peroxide stress and deacetylated by the longevity protein hSir2(SIRT1). J Biol Chem 279: 28873-28879, 2004.

40. Bertaggia E, Coletto L and Sandri M: Posttranslational modifications control FoxO3 activity during denervation. Am J Physiol Cell Physiol 302: C587-C596, 2012.

41. Senf SM, Sandesara PB, Reed SA and Judge AR: p300 Acetyltransferase activity differentially regulates the localization and activity of the FOXO homologues in skeletal muscle. Am J Physiol Cell Physiol 300: C1490-C1501, 2011.

42. Matsuzaki H, Daitoku H, Hatta M, Aoyama H, Yoshimochi K and Fukamizu A: Acetylation of Foxol alters its DNA-binding ability and sensitivity to phosphorylation. Proc Natl Acad Sci USA 102: 11278-11283, 2005

43. Ferguson D, Shao N, Heller E, Feng J, Neve R, Kim HD, Call T, Magazu S, Shen L and Nestler EJ: SIRT1-FOXO3a regulate cocaine actions in the nucleus accumbens. J Neurosci 35: 3100-3111, 2015.

44. Motta MC, Divecha N, Lemieux M, Kamel C, Chen D, Gu W, Bultsma Y, McBurney M and Guarente L: Mammalian SIRT1 represses forkhead transcription factors. Cell 116: 551-563, 2004.

45. Wang YQ, Cao Q, Wang F, Huang LY, Sang TT, Liu F and Chen SY: SIRT1 protects against oxidative stress-induced endothelial progenitor cells apoptosis by inhibiting FOXO3a via FOXO3a ubiquitination and degradation. J Cell Physiol 230: 2098-2107, 2015.

46. Wang F, Marshall CB, Li GY, Yamamoto K, Mak TW and Ikura M: Synergistic interplay between promoter recognition and $\mathrm{CBP} / \mathrm{p} 300$ coactivator recruitment by FOXO3a. ACS Chem Biol 4: 1017-1027, 2009

47. Cantó C, Gerhart-Hines Z, Feige JN, Lagouge M, Noriega L, Milne JC, Elliott PJ, Puigserver P and Auwerx J: AMPK regulates energy expenditure by modulating NAD+ metabolism and SIRT1 activity. Nature 458: 1056-1060, 2009. 